\title{
DISTRIBUIÇÃO E FREQÜÊNCIA DA EVAPOTRANSPIRAÇÃO DE REFERENCIA DE CRUZ DAS ALMAS, BA
}

\author{
Fabiano Chaves da Silva ${ }^{1}$, Carlos Ricardo Fietz ${ }^{2}$, Marcos Vinicius Folegatti ${ }^{3}$ \\ e Francisco Adriano de Carvalho Pereira ${ }^{4}$
}

\begin{abstract}
RESUMO
O objetivo deste trabalho foi estudar a distribuição de freqüência da evapotranspiração de referência na região de Cruz das Almas, BA, visando ao dimensionamento de sistemas de irrigação. Os valores máximos anuais acumulados em períodos consecutivos de 5, 10, 15, 20 e 30 dias foram ajustados às distribuições Normal, Lognormal e Beta, as quais apresentaram ajuste para todos os períodos acumulados. Por meio da distribuição acumulada Normal foram gerados valores de evapotranspiração de referência com diferentes níveis de probabilidade para a região estudada.
\end{abstract}

Palavras-chave: evapotranspiração, irrigação, probabilidade

\section{DISTRIBUTION AND FREQUENCY OF REFERENCE EVAPOTRANSPIRATION OF CRUZ DAS ALMAS, BA}

\begin{abstract}
The objective of this work was to study the distribution of probability of reference evapotranspiration for designing irrigation systems in Cruz das Almas-BA. The maximum annual values were accumulated for periods of 5, 10, 15, 20 and 30 days and were adjusted to Normal, Lognormal and Beta distribution. Through the accumulated Normal distribution, values of evapotranspiration for several probability levels were generated for the region studied.
\end{abstract}

Key words: evapotranspiration, irrigation, probability

\section{INTRODUÇÃO}

O dimensionamento de sistemas de irrigação baseia-se, geralmente, em previsões do uso de água pelas culturas. A utilização de estimativas da necessidade de água das culturas sem nenhum critério ou resultante de modelos nem sempre capazes de proporcionar resultados confiáveis, tem-se refletido, na maioria das vezes, no dimensionamento inadequado dos sistemas de bombeamento, adução, distribuição e na aplicação excedente de água (Saad, 1990).

$\mathrm{Na}$ maioria das vezes utilizam-se, como parâmetro de dimensionamento, valores máximos ou médios de evapotranspiração do período de maior exigência hídrica das culturas. Segundo Saad \& Scaloppi (1988) a grande variabilidade de valores assumidos pelos parâmetros meteorológicos durante o período de máxima exigência hídrica das culturas irrigadas, acarreta considerável dispersão dos valores calculados da evapotranspiração, sugerindo uma análise da distribuição de freqüência dos valores estimados, para fins de dimensionamento de sistemas de irrigação.

Alguns modelos para dimensionamento de sistemas de irrigação consideram o nível de probabilidade de ocorrência da evapotranspiração. Jensen (1974) apresentou um modelo de dimensionamento de sistemas que considera a probabilidade de ocorrência da evapotranspiração e precipitação pluviométrica.

$\mathrm{O}$ valor esperado da evapotranspiração que servirá de base para o dimensionamento do sistema de irrigação, depende da duração do período de máxima exigência hídrica da cultura e do nível de probabilidade desejado para as condições específicas do projeto (Pruitt et al., 1972). Segundo Jensen (1974) o período

\footnotetext{
${ }^{1}$ Eng. Agrônomo, Mestre, Doutorando, Bolsista FAPESP, DER/ESALQ/USP, CP 9, 13418-900, Piracicaba, SP, E-mail: fcdsilva@ carpaciagri.usp.br 2 Eng. Agrônomo, Dr., EMBRAPA-CPAO, CP 661, 79804-970, Dourados, MS

${ }^{3}$ Prof. Assoc., Dr. - DER/ESALQ/USP, E-mail:mvfolega@ carpa.ciagri.usp.br

${ }^{4}$ Prof. Dr. da Escola de Agronomia-UFBA, E-mail: facperei@hotmail.com
} 
de máxima exigência hídrica de culturas anuais pode variar entre duas e três semanas. Para as mesmas culturas, Saad \& Scaloppi (1988) sugerem, como razoável, admitir-se um período entre 10 e 30 dias.

O critério de escolha do nível de probabilidade deve basearse em uma análise econômica, considerando-se os prejuízos associados à redução da quantidade e da qualidade da produção, decorrentes da deficiência hídrica, e o aumento de custos do sistema para satisfazer níveis mais elevados de probabilidade. Geralmente, níveis mais elevados de probabilidade (80 a 90\%) são selecionados para culturas de grande valor econômico e condições reduzidas de água disponível no solo (Pruitt et al. 1972; Jensen, 1974). Doorenbos \& Pruitt (1977) consideram que, na maioria das regiões irrigadas, os níveis de probabilidade estejam entre 75 e $80 \%$. De acordo com Saad \& Scaloppi (1988) nas condições de irrigação suplementar dificilmente se justifica, economicamente, adotar níveis superiores a 90\%, sendo normalmente utilizados níveis que variam entre 50 a $75 \%$. O objetivo deste trabalho foi o de analisar a frequiência e a distribuição dos dados de evapotranspiração de referência, na região de Cruz das Almas, BA, e seu ajustamento aos modelos probabilísticos Normal, Lognormal e Beta.

\section{MATERIAL E MÉTODOS}

Os dados utilizados foram obtidos de uma série de 18 anos (1979 a 1996) dos registros da estação meteorológica do Centro Nacional de Pesquisa de Mandioca e Fruticultura Tropical (CNPMF/EMBRAPA) em Cruz das Almas, BA (latitude $12^{\circ}$ 40' 39" Sul, longitude 39 06' 23"' W e altitude $225 \mathrm{~m}$ ). Segundo Martorano et al. (1997) a região de Cruz das Almas está sob a influência da tipologia climática Am de Köppen. Com base em Thornthwaite, o clima é do tipo $\mathrm{C} 1 \mathrm{~s}$ A 'a que se enquadra na categoria sub-úmido com moderado excesso de água no inverno, megatérmico, em que $31 \%$ da estação de crescimento das plantas se concentram no verão.

A evapotranspiração de referência (Eto) foi estimada a partir de dados da evaporação da água no tanque classe A (ECA). As medidas de ECA ( $\mathrm{mm}$ ) foram realizadas diariamente e os seus valores foram convertidos em lâminas evapotranspiradas, por meio da Equação 1.

$$
\text { Eto }=\mathrm{Kp} . \mathrm{ECA}(\mathrm{mm})
$$

em que Kp é o coeficiente do tanque (adimensional)

Os valores do coeficiente do tanque para as condições locais de Cruz das Almas, foram obtidos com base na tabela proposta por Doorenbos \& Pruitt (1977) utilizando-se a equação de regressão ajustada por Snyder (1992):

$$
\mathrm{Kp}=0,482+0,024 \ln \mathrm{F}-0,000376 \mathrm{U}+0,0045 \mathrm{UR}
$$

em que F é a distância da área de bordadura em relação ao centro do tanque $(\mathrm{m})$, U é a velocidade do vento $\left(\mathrm{km} \cdot \mathrm{h}^{-1}\right)$ e UR é a umidade relativa do ar $(\%)$.

Os valores de Eto foram acumulados em períodos consecutivos de 5, 10, 15, 20 e 30 dias. Os valores máximos de Eto de cada respectivo ano, para todos os períodos acumulados, formaram séries de 16 dados; as distribuições teóricas de probabilidade aplicadas aos dados foram a Normal, Lognormal e Beta, conforme metodologia apresentada por Haan (1994). A média e o desvio-padrão dos valores estimados e dos logaritmos neperianos dessa variável foram definidos como as estimativas dos parâmetros de ajuste das distribuições Normal e Log normal, respectivamente, e a estimativa dos parâmetros da distribuição Beta foi obtida pelo método dos momentos (Assis et al. , 1996). A aderência dos dados às distribuições de probabilidade teóricas foi verificada através do teste de Kolmogorov-Smirnov a nível de significância de 5\%. (Campos, 1983).

\section{RESULTADOS E DISCUSSÃO}

No período de setembro a maio os valores médios mensais de Eto foram superiores a 100mm (Tabela 1). A máxima evapotranspiração foi registrada em janeiro $(170,9 \mathrm{~mm})$ e a mínima em junho $(80,2 \mathrm{~mm})$. Os coeficientes de variação calculados para as médias mensais são baixos e os desviospadrão variam de 9,6 a 34,0mm. As flutuações nos valores de evapotranspiração média mensal indicam que valores médios não são os mais adequados como indicadores da evapotranspiração de referência, sendo necessário efetuar-se uma análise de freqüência de distribuição. Analisando a Tabela 2 verifica-se que, de forma geral, a medida que diminui o período acumulado aumenta o valor médio e máximo diário da evapotranspiração de referência. Segundo Saad \& Scaloppi (1988) este comportamento ocorre, normalmente, em análise de frequiência da evapotranspiração e, dependendo do período acumulado e do nível de probabilidade adotados, pode resultar em sensíveis diferenças no dimensionamento de sistemas de irrigação.

Tabela 1. Médias mensais, desvio-padrão, coeficiente de variação e valores de máximo e mínimo mensal da evapotranspiração de referência para a região de Cruz das Almas, BA, no período 1977 e 1996

\begin{tabular}{lccccc}
\hline \multicolumn{1}{c}{ Mês } & $\begin{array}{c}\text { Média } \\
(\mathrm{mm})\end{array}$ & $\begin{array}{c}\text { Desvio-padrão } \\
(\mathrm{mm})\end{array}$ & $\begin{array}{c}\text { Coeficiente de } \\
\text { variação\% }\end{array}$ & $\begin{array}{c}\text { Máxima } \\
(\mathrm{mm})\end{array}$ & $\begin{array}{c}\text { Mínima } \\
(\mathrm{mm})\end{array}$ \\
\hline Janeiro & 170,9 & 21,2 & 12,4 & 226,3 & 142,1 \\
Fevereiro & 145,1 & 34,0 & 23,4 & 195,3 & 51,0 \\
Março & 156,6 & 21,2 & 13,5 & 200,0 & 131,3 \\
Abril & 120,7 & 16,5 & 13,6 & 152,0 & 89,7 \\
Maio & 101,1 & 16,4 & 16,2 & 136,6 & 73,5 \\
Junho & 80,2 & 9,7 & 12,1 & 101,4 & 69,1 \\
Julho & 83,5 & 9,6 & 11,5 & 104,0 & 67,2 \\
Agosto & 98,1 & 10,1 & 10,3 & 116,2 & 79,6 \\
Setembro & 116,5 & 20,7 & 17,8 & 177,2 & 89,0 \\
Outubro & 146,0 & 17,0 & 11,7 & 173,4 & 115,7 \\
Novembro & 144,2 & 13,7 & 9,5 & 168,7 & 118,1 \\
Dezembro & 168,6 & 27,9 & 16,6 & 219,8 & 92,8 \\
\hline
\end{tabular}

Tabela 2. Valores médios, máximos e mínimos observados de evapotranspiração de referência para os diversos períodos de

\begin{tabular}{|c|c|c|c|c|c|c|}
\hline \multirow{2}{*}{$\begin{array}{c}\text { Período } \\
\text { acumulado } \\
\text { dias }\end{array}$} & \multicolumn{2}{|c|}{ Média } & \multicolumn{2}{|c|}{ Valor Máximo } & \multicolumn{2}{|c|}{ Valor Mínimo } \\
\hline & $\mathrm{mm}$ & mm.dia ${ }^{-1}$ & $\mathrm{~mm}$ & mm.dia ${ }^{-1}$ & $\mathrm{~mm}$ & mm.dia ${ }^{-1}$ \\
\hline 5 & 31,57 & 6,31 & 40,70 & 8,14 & 6,49 & 1,30 \\
\hline 10 & 63,64 & 6,36 & 111,88 & 11,19 & 13,39 & 1,34 \\
\hline 15 & 92,28 & 6,15 & 136,52 & 9,10 & 13,39 & 0,89 \\
\hline 20 & 120,23 & 6,01 & 165,26 & 8,26 & 13,39 & 0,67 \\
\hline 30 & 179,98 & 6,00 & 226,35 & 7,55 & 51,05 & 1,70 \\
\hline
\end{tabular}
dias acumulados considerados 
Como se observa na Tabela 3, através do teste de Kolmogorov-Smirnov, conclui-se que as três distribuições de frequiência analisadas ajustaram-se adequadamente aos valores de evapotranspiração de referência dos períodos acumulados em estudo. Recomenda-se o uso da distribuição Normal, devido a sua maior facilidade de aplicação.

Tabela 3. Parâmetros das distribuições Normal, Lognormal e Beta para análise de frequência da evapotranspiração de referência e respectivos valores de máxima divergência (D) do teste de Kolmogorov-Smirnov*

\begin{tabular}{|c|c|c|c|c|c|c|c|c|c|}
\hline \multirow{3}{*}{$\begin{array}{c}\text { Período } \\
\text { acumulado } \\
\text { (dias) }\end{array}$} & \multicolumn{9}{|c|}{ Modelos de Distribuição } \\
\hline & \multicolumn{3}{|c|}{ Normal } & \multicolumn{3}{|c|}{ Log-normal } & \multicolumn{3}{|c|}{ Beta } \\
\hline & $\mu$ & $\sigma$ & $\mathrm{D}$ & $\mu$ & $\sigma$ & $\mathrm{D}$ & $\mathrm{p}$ & $\mathrm{q}$ & $\mathrm{D}$ \\
\hline 5 & 31,57 & 3,74 & 0,079 & 3,45 & 0,12 & 0,081 & 1,58 & 2,12 & 0,069 \\
\hline 10 & 63,64 & 12,90 & 0,156 & 4,14 & 0,16 & 0,104 & 0,56 & 2,20 & 0,126 \\
\hline 15 & 92,28 & 12,52 & 0,103 & 4,52 & 0,12 & 0,102 & 0,69 & 2,30 & 0,125 \\
\hline 20 & 120,23 & 14,53 & 0,097 & 4,78 & 0,11 & 0,095 & 1,02 & 2,36 & 0,093 \\
\hline 30 & 179,98 & 12,90 & 0,156 & 4,14 & 0,16 & 0,104 & 0,56 & 2,20 & 0,126 \\
\hline
\end{tabular}

Através da função de distribuição acumulada Normal foram gerados os valores de evapotranspiração de referência para os níveis de probabilidade de 50, 60, 70, 80, 90 e 95\% (Tabela 4). Pode-se observar, novamente, a tendência de redução da evapotranspiração diária com o aumento do período acumulado. Os níveis de probabilidade representam os limites de ocorrência de valores iguais ou inferiores aos estabelecidos; assim, por exemplo, para um período acumulado de evapotranspiração de 5 dias, existem $90 \%$ de probabilidade de que o valor da evapotranspiração não seja superior a $7,28 \mathrm{~mm} \cdot \mathrm{dia}^{-1} \mathrm{ou}$, ainda, para o mesmo período, em apenas um em cada dez anos o valor, da evapotranspiração será igual ou superior a $7,28 \mathrm{~mm} \cdot \operatorname{dia}^{-1}$. A evapotranspiração para um período acumulado de 15 dias assume valores inferiores a 7,52mm.dia ${ }^{-1}$, com $95 \%$ de probabilidade, isto é, espera-se que em 19 de cada 20 anos ocorra uma evapotranspiração de referência igual ou inferior a $7,52 \mathrm{~mm} \cdot \mathrm{dia}^{-1}$. O mesmo tipo de interpretação pode ser utilizado para se analisar todos os outros períodos acumulados.

Os valores de evapotranspiração de referência, multiplicados pelos respectivos coeficientes de cultura, poderão ser utilizados como parâmetro de dimensionamento de sistemas de irrigação

Tabela 4. Valores de evapotranspiração de referência estimados para cada período acumulado através da distribuição Normal para diferentes níveis de probabilidade e período de retorno

\begin{tabular}{|c|c|c|c|c|c|c|}
\hline \multirow{2}{*}{$\begin{array}{c}\text { Período } \\
\text { acumulado } \\
\text { (dias) }\end{array}$} & \multicolumn{6}{|c|}{$\begin{array}{c}\text { Evapotranspiração de Referência }\left(\mathrm{mm} \cdot \mathrm{dia}^{-1}\right) \\
\text { Nível de probabilidade } \%\end{array}$} \\
\hline & 50 & 60 & 70 & 80 & 90 & 95 \\
\hline 5 & 6,32 & 6,50 & 6,70 & 6,94 & 7,28 & 7,56 \\
\hline 10 & 6,37 & 6,69 & 7,04 & 7,45 & 8,20 & 8,48 \\
\hline 15 & 6,15 & 6,36 & 6,59 & 6,85 & 7,22 & 7,52 \\
\hline 20 & 6,01 & 6,20 & 6,39 & 6,62 & 6,94 & 7,20 \\
\hline 30 & 6,00 & 6,16 & 6,33 & 6,53 & 6,81 & 7,04 \\
\hline $\begin{array}{l}\text { Período de } \\
\text { Retorno (anos) }\end{array}$ & 2 & 2,5 & 3 & 5 & 10 & 20 \\
\hline
\end{tabular}

na região. Pode-se citar, como exemplo a cultura do fumo, de grande tradição na região de Cruz das Almas, BA. O período de máxima exigência hídrica ocorre 50 a 70 dias após o transplantio e o coeficiente de cultura para este estágio é 1,0 (Doorenbos \& Kassam, 1979). Nestas condições e se adotando $80 \%$ como nível de probabilidade, a evapotranspiração recomendada para o dimensionamento do sistema de irrigação para a região de Cruz das Almas seria de 6,62 mm.dia ${ }^{-1}(6,62 \times 1,00)$.

\section{CONCLUSÃO}

As distribuições de evapotranspiração de referência (Eto) acumulada ajustaram-se aos modelos de distribuições Normal, Lognormal e Beta. A evapotranspiração diária apresenta tendência de redução com o aumento do período acumulado. Os resultados obtidos poderão ser utilizados como parâmetro no dimensionamento de sistemas de irrigação na região de Cruz das Almas, BA.

\section{AGRADECIMENTOS}

Os autores agradecem ao Centro Nacional de Pesquisa de Mandioca e Fruticultura Tropical (CNPMF/EMBRAPA) pelo fornecimento dos dados meteorológicos, que serviram de base para a realização deste trabalho e como geração de importantes informações para a região.

\section{REFERÊNCIAS BIBLIOGRÁFICAS}

ASSIS F.N.. Probabilidades de ocorrência de dias sem chuva e chuvosos em Pelotas, RS. Revista Brasileira de Agrometeorologia, Santa Maria, v.2, p.87-92, 1994.

ASSIS, F.N.; ARRUDA, H.V.; PREREIRA, A.R. Aplicação de estatística à climatologia. Pelotas, Editora Universitária, Universidade Federal de Pelotas, 1996 161p.

CAMPOS, H. Estatística experimental não paramétrica. 4.ed. Piracicaba: ESALQ, 1983.

DOORENBOS, J.; KASSAM, A.H. Efectos del água sobre el rendimento de los cultivos. Roma: FAO, 1976. 194p.

DOORENBOS, J.; PRUITT, W.O. Crop water requirements. Roma: FAO, 1977. 212p.

HAAN, C.T. Statistical methods in hydrology . Ames: Iowa State University Press, 1994. 378p.

JENSEN, M.E. Consumptive use of water and irrigation water requirements. New York: ASCE, 1974. 215 p.

MARTORANO, L.G.; PEREIRA, F.A.C.; SANTOS, C.A.S; PEREIRA, A.R.; VILLA NOVA, N.A. Análise das condições climáticas na região de Cruz das Almas, Bahia. In: CỎNGRESSO BRASILEIRO DE AGROMETEOROLOGIA, 10., 1997. Piracicaba, SP. Anais .... Piracicaba: v. 1, p. $262-264$.

PRUITT, W.O.; von OETTINGEN, S.; MORGAN, D.L. Central California evapotranspiration frequencies. Journal of the Irrigation and Drainage Division, New York, v. 98, n. IR2, p. 177-184, 1972.

SAAD, J.C.C. Estudo das distribuições de frequiência da evapotranspiração de referência e da precipitação pluvial para fins de dimensionamento de sistemas de irrigação. Piracicaba: ESALQ, 1990 124p. (Dissertação Mestrado)

SAAD, J.C.C.; SCALOPPI, E.J. Freqüência de distribuição de evapotranspiração para dimensionamento de sistemas de irrigação. In: CỎNGRESSO NACIONAL DE IRRIGAÇÃO E DRENAGEM, 8., 1988. Florianópolis, SC. Anais... Florianópolis: ABID, v. 2, p. 1037-1052.

SNYDER, R.L. Equation for evaporation pan to evapotranspiration conversions. Journal of Irrigation and Drainage Engineering 118:977, 1992 